\title{
Determinants of Pulse Rate change and Time-To Default from Treatment among Congestive Heart Failure Patients in Felege- Hiwot Referral Hospital, Bahir Dar, Ethiopia; comparison of separate and joint models
}

Yikeber Abebaw Moyehodie ( $\nabla$ ykebera@gmail.com )

Debre Tabor University

Kasim Mohammed Yesuf

University Of Gondar

Adem Aragaw Sied

Mizan Tepi University

Bezanesh Melese Masresha

Debre Tabor University

\section{Research Article}

Keywords: Pulse Rate, Congestive Heart Failure, Time to Default, Survival Analysis, Longitudinal Analysis, Proportional Hazard Model, Linear Mixed Model, Joint Model

Posted Date: March 12th, 2021

DOI: https://doi.org/10.21203/rs.3.rs-228538/v1

License: (c) (i) This work is licensed under a Creative Commons Attribution 4.0 International License. Read Full License 


\section{Abstract}

Background: Globally, heart failure is a rapidly growing public health issue with an estimated prevalence of $>37.7$ million individuals. It is a shared chronic phase of cardiac functional impairment secondary to many etiologies. The main purpose of this study was to identify factors that affect the longitudinal changes of pulse rate and survival endpoints, time-to default among Congestive Heart Failure Patients in Felege- Hiwot Referral Hospital, Bahir Dar, Ethiopia.

Methods: Hospital based retrospective studies were conducted among 302 congestive heart failure patients who were 15 years old or older and who were on treatment follow-up from the first February 2016 to thirty-one December 2018 in Felege-Hiwot Referral Hospital, Bahir Dar, Ethiopia. First, data were analyzed using linear mixed model and survival models separately, and then the joint models of both submodels were analyzed by linked their shared unobserved random effects using a shared parameter model.

Results: Out of the total $302 \mathrm{CHF}$ treatment followers, 103 (34.1\%) of the patients were defaulting from treatment. The mean pulse rate of female and male patients was 87.25 and 90.20 , respectively. Averagely $51.12 \%$ of blood in the left ventricle is pushed out with each heartbeat. The results for separate and joint models were quite similar to each other but not identical. However, the estimated association parameter (a) in the joint model is $(\mathrm{HR}=1.0311,95 \% \mathrm{Cl}: 1.0033,1.0597, \mathrm{P}=0.0278)$, providing there is evidence of a positive association between the survival and the longitudinal sub-models. Thus, defaulting is more likely to occur in patients with higher pulse rates. Patients, being male, hypertensive, CKD, pneumonic, and NYHA class IV patients were associated with a higher risk of defaulting. Age, LVIF, follow-up time in a month had a negative significant effect and NYHA class, and male gender had a positive significant effect on average evaluation of pulse rate of patients.

Conclusions: The patient who are male, NYHA class IV, had low LVIF and comorbid with hypertensive, CKD, pneumonia were risk factors of pulse rate change and defaulting from treatment of CHF patients. The joint model was preferred for simultaneous analyses of repeated measurement and survival data.

\section{Introduction}

Globally, heart failure is a rapidly growing public health issue with an estimated prevalence of > 37.7 million. It is a shared chronic phase of cardiac functional loss secondary to many etiologies. In the USA, the total medical costs for patients with HF are expected to rise from US\$20.9 billion in 2012 to $\$ 53.1$ billion by 2030 [1]. Over 1 million individuals within the UK have HF, with prevalence rates expected to extend over subsequent decade. This places a big financial burden on the United Kingdom [2].

In Africa, $7-10 \%$ of all medical admissions to the hospital had cardiac diseases, out of this $3-7 \%$ had heart failure. Compared with the developed world Africa has the highest proportion of HF patients. In Africa heart failure has a higher effect on the health and health economics [3]. 
In Sub Saharan Africa, HF is the main public health concern and is associated with high morbidity and mortality, high rates of recurrent hospitalization, poor quality of life, and loss of economic productivity, as it affects mostly young and economically active adults. However, due to economic limitations, it is difficult challenging for most HF patients in SSA to get the essential treatment. They get difficulty to preserve regular follow-up visits as a result cumulative premature deaths have occurred [4].

In Ethiopia, approximately 9\% of all deaths were caused by CHF according to WHO [5] report. CHF has a cumulative problem, especially in urban settings in Ethiopia. It is one of the prevalent causes of morbidity ranging from 4 to $24 \%$, and mortality ranging from 6.5 to $24 \%$. It is highly prevalent among older population, and the leading causes of medical intensive care unit admission ranging from 8.9 to $9.8 \%$. In Addis Ababa, averagely $25 \%$ of all household deaths, and $11 \%$ of all hospital deaths were due to CHF [5]. In Felege-Hiwot Referral Hospital HF is a progressive public health problem with high morbidity and mortality[6].

The heart rate/pulse rate is the number of times the heart beats in one minute and it is used to know whether abnormal or irregular pulse rate, or heart block occurred or not. The normal resting heart rate is nearly 60 to 80 beats per minute [7]. There are not common body parts of a patient for taken heart rate measurement [8]. It can be measured in different parts of the body, but the two most common parts of the body are the wrist and the neck [9]. Epidemiological and randomized clinical trial studies are focused on the predictive value of heart rate. It proposes that a continued increment in heart rate increases the progression as well as the severity of the disease [10]. A greater heart rate measure is associated with a high risk of HF hospitalization and an inferior pulse rate measure at the base line and through follow-up time is positively related with minor risk of mortality and re-hospitalization [11].

Although the advancement in therapy and treatment of heart failure is high, nowadays it is a chronic illness with an unpromising diagnosis, and has a high socioeconomic problem [12]. HF is one of the major causes of hospitalization and high rates of mortality in several countries [13]. Even though the number of HF patients admitted to CHF clinics is increasing from year to year, there are a number of HF related deaths and loss to follow-ups every year. This indicates that there are other factors affecting the progression of the disease and the survival status of CHF patients as well. Therefore, it is essential to assess risk factors associated with the effectiveness of the treatment over time. Most researchers conducted their study in relation to heart failure patients using cross-sectional study, survival time of the patients after they admitted to CHF clinic, and linear mixed models. However, Seid, Getie [14] suggests that these linear mixed effect model analysis for longitudinal data, and Weibull or semi-parametric (Cox) proportional hazard model analysis for survival data separately were inappropriate when the longitudinal variable is correlated with patient health status (survival time), because of the survival endpoint occurrences, as well as the possibility of study dropout. Moreover, Grover, Swain [15] studied the joint modeling of both longitudinal and survival data analysis, containing all information simultaneously, and gives lawful and effective inferences. Therefore, it is usually recommended to jointly model the longitudinal data analysis and survival data analysis altogether via a shared random effects model in 
order to account for the dependence between longitudinal and survival components on the same subject and any available covariates.

To the best of our knowledge, there is no research conducted on joint model analysis of longitudinal pulse rate and survival end point time to default from $\mathrm{CHF}$ treatment and no research conducted on survival analysis of time to default from treatment of $\mathrm{CHF}$ patients. However, defaulting remains a public health problem which needs to be addressed. A person who was defaulting from congestive heart failure treatment is at risk for clinical worsening and difficulties including deterioration in drug opposition and death. Hence, we aimed to identify factors that affect the longitudinal changes in pulse rate and survival endpoints, time-to default among Congestive Heart Failure Patients in Felege- Hiwot Referral Hospital.

\section{Methods}

\subsection{Study Design, and Area}

This study was carried out by using a retrospective cohort study design based on data obtained from Felege-Hiwot Referral Hospital, Bahir-Dar, Amhara, Ethiopia. The Amhara Regional state is located in the northwestern part of Ethiopia. It is located $563 \mathrm{~km}$ far from Addis Ababa, the capital city of Ethiopia. Felege-Hiwot Referral Hospital is a teaching and referral hospital and one of the oldest hospitals in Ethiopia. It is a tertiary health care labeled hospital serving the people of Bahir Dar town and remote area populations as Teaching and Referral Hospital; delivering health services to their dependents (patients), as well as public patients referred by other specialized hospitals.

\subsection{Study period, and Source of Data}

In this study, we used secondary sources of data. The data had been obtained from Amhara region at FHRH Outpatient Department (OPD) in CHF clinic during the follow-up time of 1st February 2016 to 31th December 2018. It is extracted from the patients' chart which contains epidemiological, laboratory, and clinical information of all CHF patients under follow-up, including a detailed heart failure history and socio-demographic variables. The data was collected by health care service providers of CHF clinic after we had given adequate orientation to them about the way of data collection and the variables that were included in the study.

\subsection{Study Population}

Patients whose age 15 years old and above attend at least for 8 months (a minimum of two follow- up visits) for refilling their prescription, and who are initiated on treatment during the study period at FHRH were included in the study. Therefore, among the total of $1334 \mathrm{CHF}$ patients registered from 1 st February 2016 to 31 th December 2018, only 302 CHF patients satisfy the inclusion criteria and hence are included in this study.

\subsection{Study Variable}

The two response variables considered for this study were the longitudinal measured pulse rate - number of heart beats per minute measured every 4 months irrespective of their visit to $\mathrm{CHF}$ patient clinic and the 
survival outcome - time to default from treatment for CHF patients. Defaulters used in this study were those patients who missed contact for at least eight months (who had missed two or more clinical appointments), which may be due to death, loss to follow, or transferring to another hospital.

\section{Explanatory Variables}

Covariates associated with repeated measure pulse rate and time to default were gender (female, male), residence (rural, urban), age, type of $\mathrm{CHF}$ (left side failure, right side failure, biventricular failure), etiology of heart failure(VHD,HHD, IHD, cor pulmonale, dilated cardiomyopathy, other etiology's), NYHA class (class II, class III, class IV), left ventricle ejection fraction, observation time, weight, presence of diabetes mellitus (no, yes), presence of hypertension (no, yes), presence of pneumonia (no, yes), presence of chronic kidney disease (no, yes), presence of anemia (no, yes).

\subsection{Data Processing and Analysis}

In this study, different statistical analysis including both descriptive and inferential statistics were used. The researcher analysis, a separate model for each longitudinal and survival model, and a joint model of longitudinal and survival analysis for longitudinal measurements and time-to-event outcome of CHF patients linked by subject-specific random effects. Data management has been done by SAS 9.4 and R version 3.5.3 statistical software. SPSS version 23.0 has been used for data entering. Statistical decision was made at $5 \%$ level of significance.

\section{Results And Discussion}

\subsection{Descriptive statistics}

Descriptive statistics were used to summarize the characteristics of participants in the study area. The characteristics of the respondents were summarized as indicated in Table 1. Out of the total 302 patients, $168(55.6 \%)$ were females and the remaining 134(44.4\%) were males. Majority of the CHF patients 213 $(70.5 \%)$ were rural residents. Of those of the CHF patients, 118 (39.1\%) had biventricular heart failure, 84 $(27.8 \%)$ had faced left-sided heart failure, and the rest 100 (33.1\%) had faced right-sided heart failure. For the reason that $\mathrm{HF}$ is not a disease by itself, patients with $\mathrm{HF}$ have other causes, out of this cause of heart failure for patients, $96(31.8 \%)$ were by VHD, $42(13.9 \%)$ were by HHD, 26(8.6\%) were by IHD, 35(11.6\%) were by cor-pulmonale, $66(21.9 \%)$ were by dilated cardiomyopathy and the remaining $37(12.3 \%)$ of patients were by other etiology's. When we observed the NYHA class of CHF patients, $50(16.6 \%)$ of them were NYHA class II, 106(35.1\%) were class III, 146(48.3\%) were class IV. Furthermore, from the comorbidities of heart failure patients, 73 (24.2\%) were having hypertension, 36(11.9\%) were with Diabetes Mellitus, 71(23.5\%) were with Pneumonia, 46(15.2\%) were with coronary kidney disease and 63(20.9\%) were had anemia.

Out of the total $302 \mathrm{CHF}$ treatment followers, about $55.2 \%$ male respondents are defaulting from treatment and the remaining are censored. On the other hand, about $17.3 \%$ female respondents were default and the remaining respondents were censored. Based on the patient's residence, out of 213 rural 
and 89 urban patients, $38.55 \%$ and $23.6 \%$ of the respondents had an occurrence of an event, respectively. When we saw NYHA class of CHF patients, NYHA class II $14.0 \%$, NYHA class III $20.8 \%$, and NYHA class IV $50.7 \%$ respondents were default. The mean pulse rate of female and male patients was 87.25 and 90.20 , respectively. The remaining variables are described in the same way.

The mean of baseline age, weight, and left ventricular ejection fraction of patients were 47.88 years (with a standard deviation of 19.116 years), 56.95 kilograms (with a standard deviation of 8.239 kilograms) and 51.12 percent (with a standard deviation of 15.02 percent), which means that averagely 51.12 percent of blood in the left ventricle is pushed out with each heartbeat respectively.

\subsection{Separate Analysis of Longitudinal Data}

As can be observed from Table 2 below, the analysis of the longitudinal data was based on linear mixed effects model incorporates patient-specific pulse rate variability. In this model, among the six predictors included in the model, age, gender, LVEF, NYHA Class IV, and follow-up time were statistically significant. The random effect part of the above table (Table 3 ) indicates how the evolution of pulse rate for the $i^{\text {th }}$ subjects deviates from the average evolution in the population. The results showed that the variance and covariance of the random effects are significantly different from zero. This indicates that the pulse rate values at baseline vary across subjects and the change of pulse rate measured over time varies between subjects.

\subsection{Separate Analysis of Survival Data}

Among the candidate variables considered for building multivariable Cox, the stepwise procedure picked up six variables, weight, gender, NYHA class, presence of hypertension, presence of pneumonia, and presence of coronary kidney disease. The proportional hazards assumption asserts that the hazard ratios are constant overtime. That means the risk of failure must be the same no matter how long the subjects have been followed.

The results of the separate survival models are presented in Table 3. In this separate analysis of cox regression model, the gender of CHF patients, NYHA class (IV) patients, patients who had hypertension, pneumonia, and CKD were positively associated with defaulting time. However, the weight of patients was negatively associated with defaulting time for treatment of $\mathrm{CHF}$ patients at a $5 \%$ level of significant.

\subsection{Joint Model of Survival and Longitudinal Analysis}

In many clinical studies, subjects are followed up repeatedly and response data collected, and this response data may be censored by time-to-event outcome. A common objective in follow-up studies is to characterize the relationship between longitudinal measurements and time-to-event outcomes. If the two outcome processes are correlated, modelling the longitudinal and event-time outcomes separately can be inefficient and can lead to biased estimation. To alleviate these problems, we fit the joint model approach for longitudinal (pulse rate measure) and time-to-event (time to default from treatment) data, to identify predictors that affect the two responses and to check whether there is an association between 
unobserved longitudinal outcome (PR) and time to default of CHF patients. The result of the joint model could be obtained by combining the selected random-intercept-and slope model and Cox-proportional hazard model.

Table 4 displays the results of joint modeling for longitudinal and survival process, the result shows that the predictor age, LVEF, gender, NYHA class, and observation time was significantly associated with average PR. And, the result shows that the predictors gender, hypertension, CKD, pneumonia, and NYHA class were significantly associated with time to default of CHF patients in the survival sub model. The estimate of the association parameter (a) is positive (0.0307), indicating that pulse rate is positively associated with the risk of default of patients to heart failure treatment. This means that a decreasing trend in the pulse rate of patients undergoing heart failure treatment significantly reduces the risk of defaulting for those patients. Moreover, the estimate of the association parameter in the joint analysis is significantly different from zero, providing strong evidence of association between the effects of the longitudinal outcome on the risk of an event. We also observe that the random effect parameter estimates for joint model analysis. It shows that the variation of the random intercepts was higher than the random slope, which implies higher baseline difference. The output also displays the correlation coefficient between the random intercept and the random slope, which indicates that the slope of time and intercept are dependent because of the dependence of the slope and the intercept of each individual. Therefore, the variability between patients in intercept was 100.2722 , the variability between patients in slope was 0.1219 , the correlation between intercepts and slope were -0.5468 , which indicates that there is a negative correlation between the intercept and slope of linear time effect for the random part and the variability within patients were 120.7801 .

\subsection{Comparison of Separate and Joint Models}

In both the longitudinal submodel and separate longitudinal model follow-up times, age, gender, LVEF, and NYHA class IV were statistically significant predictors. However, the longitudinal submodel has a narrow confidence interval, which indicates its standard error is small for all significant predictors compared to the separate models. In the survival submodel of the joint model, except weight, all predictors included in the model were significantly associated with the hazard of defaulting. When evaluating the overall performance of both the separate and joint models in terms of model parsimonious and goodness of fit, the joint model was preferred as it has a smaller total AIC than the separate model. Moreover, the statistical significance of the association parameter is evidence that the joint model is better than the separate models [14]. As (Table 4) revealed, the estimate of the association parameter in the survival submodel analysis under the joint model was significantly different from zero, this indicates that the two outcomes are correlated. Therefore, the joint model was found preferable to fit the data better over the separate one.

\subsection{Interpretation and Discussion of the Results}


In this study, three different models were explored, the linear mixed effects model, a cox proportional hazard model for each outcome independently, and joint modeling of the two outcomes together (i.e. longitudinal and survival). Since joint model building usually starts from separate models for each component, initially each data was analyzed separately. Such a separate analysis is preferred for several reasons. Firstly, it helps to specify the mean response of the model. Secondly, the random effects to be included in the longitudinal model can be easily determined, and thirdly, the initial values to be provided for the joint models can be obtained.

After the most suitable separate models had been decided for the data, the proposed joint models were applied to the data, with the aim of investigating the effects of repeated measure pulse rate measurements on time-to-default. In the longitudinal submodel, age, left ventricular ejection fraction, follow-up time, gender, and patients with NYHA class IV are statistically associated with the progression of pulse rate. Increment in the progression of pulse rate over follow-up time indicates the severity of the disease, whereas the decrease in the evolution of the longitudinal measured pulse rate indicates the effectiveness of the treatment (reduction in severity of the disease). In this study, follow-up time had a significant negative effect on the average evolution of pulse rate. Patients with more follow-up time made a particular decrease in pulse rate $(P<0.0001)$. Which means follow-up time in months reduces the severity of the disease.

When the age of a patient increases by one year, the average pulse rate of patients is decreased by $8.88 \%$, while all other predictors are held constant $(P=0.0040)$. This finding is in line with other studies $[7,11$, 16], which showed that ages were found to be negatively associated with the average evolution of PR.

When the left ventricle ejection fraction of patients is increased by one unit, the average pulse rates of patients are decreased by $11.10 \%$, while all other predictors are held constant $(P=0.0043)$. This means that if pushed out blood in the left ventricle in each heart beat increased by one percent, the average pulse rate of patients deceased by $11.10 \%$. This finding is in line with other studies [7, 11], which showed that LVEF were found to be negatively associated with the average evolution of pulse rate. However, our finding contradicts with the earlier study by Nesbitt, Doctorvaladan [17] which shows that no evidence of an association between left ventricular ejection fraction and quality of life of HF patients.

Among New York heart association classes (NYHA class), NYHA class IV patients were found to have a significant difference compared to NYHA class II patients $(P=0.0442)$. This implies that the progression of the average pulse rate of NYHA class IV patients starting CHF treatment were higher by 3.3619 than those NYHA class II patients. This finding is in line with other studies [7], which showed that NYHA class had positively significantly associated with PR. Moreover, this finding is in line with the previous finding by Nesbitt, Doctorvaladan [17] which shows that Higher NYHA class reduced the quality of life of CHF patients.

The average pulse rate of male patients is greater by 2.5063 compared to female patients, while other covariates are kept constant $(P=0.0335)$. This result also conforms to the result of Nesbitt, Doctorvaladan [17], which shows that male gender is associated with reduced quality of life of CHF 
patients. However, our study contradicts earlier studies [7, 16], which showed that female patients had a higher pulse rate than males during the follow-up. However, in our finding gender male is having a higher significant effect in the evolution of the average pulse rate of patients compared to female patients.

In the survival submodel, gender, male, hypertension, CKD, pneumonia, and NYHA class IV are significantly associated with the hazard of defaulting from treatment. Being male patients were associated with a risk of default ( $\mathrm{HR}=3.8958,95 \% \mathrm{Cl}: 2.4771,6.1272, \mathrm{P}=<.0001)$ that were male patients had 3.8958 times high risk of default compared to female patients holding others covariates in the model constant. This result was supported by most of the studies [18], which showed female patients had a slightly higher survival probability than male patients. This study were also in line with the studies [19] and [20], which indicates that male gender had a significant effect on mortality among heart failure patients. However, our study contradicts with the earlier study [21], which showed that no significant differences in mortality among gender of heart failure patients.

The estimated risk of defaulting for a patient with NYHA class (IV) compared to NYHA class(II) patients is $(\mathrm{HR}=4.1152,95 \% \mathrm{Cl}: 1.6251,10.4210, \mathrm{P}=0.0028)$ indicating that the hazard rate of defaulting from treatment for NYHA class (IV) patients is around four times higher than NYHA class (II) patients, holding others covariates in the model constant. This result confirms to the result obtained by [19], which indicates that higher NYHA class patients had a higher risk of death.

The estimated risk of defaulting for a heart failure patient with the presence of hypertension is $(\mathrm{HR}=$ $2.7563,95 \% \mathrm{Cl}: 1.8265,4.1594, \mathrm{P}=<.0001)$, This indicates that the risk of defaulting for hypertensive patients were 2.7563 times higher compared to non-hypertensive patients keeping other variables constant. This finding is in line with the previous findings [22]and [6], which showed hypertension was positive and significantly associated with the prevalence of HF. Likewise, estimated risk of defaulting for CHF patients with presence of Pneumonia and CKD as co morbidity (HR $=1.6449,95 \%$ Cl: 1.0895, 2.4833, $\mathrm{P}=0.0179),(\mathrm{HR}=1.7762,95 \% \mathrm{Cl}: 1.0906,2.8930, \mathrm{P}=0.0210))$ respectively. This indicates that the risks of defaulting for pneumonic and CKD patients were 1.6449 and 1.7762 times the risk of defaulting for a patient who had no pneumonia, and CKD respectively keeping other variables constant.

Furthermore, the estimated value for the association parameter $(a)$ in the joint model is 0.0307 and the hazard ratio is 1.0311 (with $95 \% \mathrm{Cl}: 1.0033,1.0597$ ) and statistically significant ( $p$-value $=0.0278$ ). This indicates that there is strong evidence of association between the effects of the longitudinal biomarker to the risk of default. For a unit change in the true value of the repeated measure pulse rate, the relative change in the risk of defaulting is 1.0311 while other variables are kept constant. The results indicate that lower values of pulse rate are associated with better survival, which is in line with the findings [11, 16, 23], higher resting heart rate was predictive of earlier mortality and heart rate reduction is associated with improved clinical outcomes in heart failure patients, which is in line with the findings [11, 16, 23], higher resting heart rate is predictive of earlier mortality and heart rate reduction is associated with improved clinical outcomes in heart failure patients. This finding contrasts with the previous finding [24] which 
showed that a joint effect of both the mean number of abnormal heart rate (pulse rate) measurements and the risk of re-hospitalization were not statistically significant $(p=0.1650)$.

\section{Conclusion}

The study investigated and identified factors that are associated with the longitudinal measure PR and defaulting time of CHF patients from treatment in Felege-Hiwot referral hospital using both separate and joint model analysis. For this specific study, the joint model was the better fit than the separate survival and longitudinal models. Both separate longitudinal and longitudinal submodels showed that: age, LVEF, follow-up time of the patients, gender, and NYHA class were significant predictors of the progression change of pulse rate. The variables age, LVEF, and follow-up time were negatively associated, whereas male gender and NYHA class were positively associated with the progression change of pulse rate of patients. Since follow-up time of patients had a negative impact, we conclude that the rate of progression of pulse rate decreases over time. Moreover, the separate survival model analysis showed that: male gender, CKD, hypertension, pneumonia, NYHA class positively associated, and weight negatively associated with the time to default. And in the survival submodel CKD, hypertension, pneumonia, NYHA class, male gender, and unobserved true PR were positively associated with the time to default. When evaluating the overall performance of both the separate and joint models in terms of model parsimony, goodness of fit, smaller total AIC, and the statistical significance of association parameters, the joint model performs better. Thus, we concluded that the joint model was preferred for simultaneous analyses of repeated measurement and survival data.

\section{Limitation}

A limitation of the study is that since the information on some important variables such as body mass index, alcoholism, marital status, education level, and smoking status are not available in the patients' charts, we have not used such variables in our research.

\section{Abbreviations}

AIC: Akaike's Information Criterion; CHF; Chronic or Congestive Heart Failure: Cl; Confidence Interval: CKD; Chronic Kidney Disease: FHRH; Felege-Hiwot Referral Hospital: HF; Heart Failure: HR: Hazard Ratio: LVEF; Left Ventricular Ejection Fraction: NYHA; New York Heart Association: PR; Pulse Rate: RHD; Rheumatic Heart Disease: SPSS; Statistical Package for Social Science: Std.Dev; Standard Deviation: SE; Standard Error: SSA; Sub-Saharan Africa: VHD; Valvular Heart Disease: WHO; World Health Organization

\section{Declarations}

Ethical approval and consent to participate: The data used in the current investigation was collected previously by the health staff for treatment purpose/for diagnosis heart failure and to start follow-up treatment. To use this previously collected data, an ethical approval certificate had been obtained from 
University of Gondar Ethical Approval Committee, University of Gondar, Ethiopia, with reference number: RCS/381/2019. In data collection, there was no written or verbal consent from participants. The reason was the investigators didn't get participants rather, and secondary data was obtained in the patients' chart. The Ethical approval committee approved for the use of this secondary data for the current investigation.

Consent for publication: Not applicable

Availability of data and materials: The authors confirmed that the data used for this research is available from the corresponding author.

Competing interests: The author(s) declare(s) that they have no competing interests.

Funding: Not applicable.

Authors' contributions: YK initiated the research and involved in the write-up and development of the proposal, the data collection format, data entry, and data analysis, and write-up of the manuscript. KM, $\mathrm{BM}$ and $\mathrm{AA}$ participated in design and data analysis and critically read the manuscript and amended constructive comments for the betterment of the manuscript applying his rich experience. All authors read and approved the final version of the manuscript.

\section{Acknowledgements}

My special gratitude goes to doctor Getaneh Kassie, my best friend, and Felege-Hiwot Referral Hospital staff member for providing important information and unreserved support. I would like to express my special thanks to all participants and data collactors.

\section{References}

1. Ziaeian, B. and G.C. Fonarow, Epidemiology and aetiology of heart failure. Nature Reviews Cardiology, 2016. 13(6): p. 368.

2. Cook, C., et al., 49 The Annual Global Economic Burden of Heart Failure. Heart, 2014. 100(Suppl 3): p. A28-A29.

3. Ntusi, N.B. and B.M. Mayosi, Epidemiology of heart failure in sub-Saharan Africa. Expert review of cardiovascular therapy, 2009. 7(2): p. 169-180.

4. Agbor, V.N., et al., Heart failure in sub-Saharan Africa: a contemporaneous systematic review and meta-analysis. International journal of cardiology, 2018. 257: p. 207-215.

5. WHO, Global status report on noncommunicable diseases 2014. 2014, World Health Organization.

6. Zeru, M.A., Assessment of major causes of heart failure and its pharmacologic management among patients at Felege Hiwot referral hospital in Bahir Dar, Ethiopia. Journal of Public Health and Epidemiology, 2018. 10(9): p. 326-331. 
7. Fissuh, Y.H. and G. Muletav, A joint model for a longitudinal pulse rate and respiratory rate of congestive heart failure patients: at Ayder Referral Hospital of Mekelle University, Tigray, Ethiopia. J Biom Biostat, 2015. 6(5): p. 1.

8. Greene, S.J., et al., The prognostic significance of heart rate in patients hospitalized for heart failure with reduced ejection fraction in sinus rhythm: insights from the EVEREST (Efficacy of Vasopressin Antagonism in Heart Failure: Outcome Study With Tolvaptan) trial. JACC: Heart Failure, 2013. 1(6): p. 488-496.

9. Aarts, L.A., et al., Non-contact heart rate monitoring utilizing camera photoplethysmography in the neonatal intensive care unit-A pilot study. Early human development, 2013. 89(12): p. 943-948.

10. Custodis, F., et al., Vascular pathophysiology in response to increased heart rate. Journal of the American College of Cardiology, 2010. 56(24): p. 1973-1983.

11. Kurgansky, K.E., et al., Association of pulse rate with outcomes in heart failure with reduced ejection fraction: a retrospective cohort study. BMC cardiovascular disorders, 2020. 20(1): p. 1-11.

12. Farmakis, D., et al., The medical and socioeconomic burden of heart failure: a comparative delineation with cancer. 2016, Elsevier.

13. Wajner, A., et al., Causes and predictors of in-hospital mortality in patients admitted with or for heart failure at a tertiary hospital in Brazil. Arquivos brasileiros de cardiologia, 2017(AHEAD): p. 0-0.

14. Seid, A., et al., Joint modeling of longitudinal CD4 cell counts and time-to-default from HAART treatment: a comparison of separate and joint models. Electronic Journal of Applied Statistical Analysis, 2014. 7(2): p. 292-314.

15. Grover, G., et al., A Joint Modeling Approach to Assess the Impact of CD4 Cell Count on the Risk of Loss to Follow up in HIV/AIDS Patients on Antiretroviral Therapy. 2015.

16. CASTAGNO, D., et al. Heart rate and outcomes in a broad spectrum of patients with chronic heart failure: results from the CHARM programme. in Heart Failure Congress 2011. 2011.

17. Nesbitt, T., et al., Correlates of quality of life in rural heart failure patients. Circulation: Heart Failure, 2014: p. CIRCHEARTFAILURE. 113.000577.

18. Hailay, A., E. Kebede, and K. Mohammed, Survival During Treatment Period of Patients with Severe Heart Failure Admitted to Intensive Care Unit (ICU) at Gondar University Hospital (GUH), Gondar, Ethiopia. 2015.

19. Barlera, S., et al., Predictors of mortality in 6975 patients with chronic heart failure in the Gruppo Italiano per lo Studio della Streptochinasi nell'Infarto Miocardico-Heart Failure trial: proposal for a nomogram. Circulation: Heart Failure, 2013. 6(1): p. 31-39.

20. Zomer, A.C., et al., Heart failure admissions in adults with congenital heart disease; risk factors and prognosis. Int J Cardiol, 2013. 168(3): p. 2487-93.

21. Ahmad, T., et al., Survival analysis of heart failure patients: A case study. PloS one, 2017. 12(7): p. e0181001. 
22. Beck, H., et al., Heart failure in a cohort of patients with chronic kidney disease: the GCKD study. PloS one, 2015. 10(4): p. e0122552.

23. Kupper, N., et al., Cardiovascular reactivity to mental stress and mortality in patients with heart failure. JACC: Heart Failure, 2015. 3(5): p. 373-382.

24. Njagi, E.N., Joint Models for Survival and Longitudinal Data, Missing Data, and Sensitivity Analysis, with Applications in Medical Research. 2013.

\section{Tables}

Due to technical limitations the tables are available as a download in the supplemental files.

\section{Supplementary Files}

This is a list of supplementary files associated with this preprint. Click to download.

- Tables.pdf 